\title{
Midterm results of endovascular treatment of atherosclerotic aneurysms of the descending thoracic aorta
}

Bertrand Marcheix, MD, ${ }^{a}$ Camille Dambrin, MD, PhD, ${ }^{a}$ Jean-Philippe Bolduc, MD, ${ }^{\text {b }}$ Catherine Arnaud, MD, ${ }^{c}$ Christophe Cron, MD, ${ }^{a}$ Lucy Hollington, MD, ${ }^{d}$ Antoine Mugniot, MD, ${ }^{a}$ Philippe Soula, MD, ${ }^{a}$ Mehdi Bennaceur, MD, Valérie Chabbert, MD, ${ }^{b}$ Pierre Massabuau, MD, ${ }^{e}$ Philippe Otal, MD, ${ }^{b}$ Alain Cérène, $\mathrm{MD}^{\mathrm{a}}$ and Hervé Rousseau, MD, $\mathrm{PhD}^{\mathrm{b}}$

From the Departments of Thoracic and Cardiovascular Surgery, ${ }^{\mathrm{a}}$ Radiology, ${ }^{\mathrm{b}}$ and Cardiology, ${ }^{\mathrm{e}}$ Rangueil University Hospital, Toulouse, France; the Department of Epidemiology and Biostatistics, ${ }^{c}$ Toulouse University Hospital, Toulouse, France; and the Department of Cardiology, ${ }^{\mathrm{d}}$ Montauban General Hospital, Montauban, France.

Received for publication March 4, 2006; revisions received June 24, 2006; accepted for publication July 7, 2006

Address for reprints: Hervé Rousseau, MD, Radiology Department, Rangueil University Hospital, TSA 50032, 1 Avenue Jean Poulhès, 31059 Toulouse, Cedex 9, France (E-mail: rousseau.h@chu-toulouse.fr).

J Thorac Cardiovasc Surg 2006;132:1030-6 $0022-5223 / \$ 32.00$

Copyright () 2006 by The American Association for Thoracic Surgery

doi:10.1016/j.jtcvs.2006.07.006
Objective: We sought to determine the midterm results of endovascular repair of atherosclerotic aneurysms of the thoracic descending aorta by using second-generation, commercially available stent grafts.

Methods: Between 1996 and 2005, 45 patients (mean age, $68 \pm 11$ years) with aneurysms of the descending thoracic aorta underwent endovascular repair. Aortic dissections, penetrating ulcers, and traumatisms were excluded. The mean follow-up was $24.7 \pm 21.6$ months (maximum, 6.7 years).

Results: No patients died, and no conversion to surgical intervention was required during the procedures. Three (6.7\%) patients died during the first month, and 6 $(14.7 \%)$ died later on. The main complications were strokes (13.3\%), vascular access complications (8.9\%), aortic complications (6.6\%), paraplegia (4.4\%), and sudden deaths $(4.4 \%)$. Nineteen $(42 \%)$ primary endoleaks were encountered: 3 required reinterventions, 15 spontaneously thrombosed, and 1 patient died. Except for 2 sudden unexplained deaths, no aortic complications were observed after 1 month. Actuarial survival estimates at 1,3 , and 5 years were $87.6 \% \pm 5.3 \%, 76.9 \% \pm 7.4 \%$, and $70.6 \% \pm 9.2 \%$, respectively. Actuarial freedom from death related to the treated aortic disease was $94.3 \% \pm 4.0 \%, 94.3 \% \pm 4.0 \%$, and $86.4 \% \pm 8.4 \%$ at 1,3 , and 5 years, respectively. Aspirin status of greater than $3(P=.005)$, high aortic diameter $(P=.007)$, and long covered lengths $(P=.02)$ were determinant for mortality. Actuarial freedom from complication was $62.6 \% \pm 7.7 \%, 58.9 \% \pm$ $8.1 \%$, and $58.9 \% \pm 8.1 \%$ at 1,3 , and 5 years, respectively. The location of the aneurysm $(P=.05)$ and a high aortic diameter $(P=.04)$ were both determinants for endoleaks.

Conclusions: Stent grafting of atherosclerotic aneurysm of the thoracic descending aorta is safe and effective. Further studies are mandatory to determine the most relevant indications and the long-term efficacy of such treatment.

$\mathrm{T}$ he second half of the 20th century produced significant advances in the management of patients with aneurysms of the descending thoracic aorta, and traditional treatment consisted of open surgical graft replacement. Many open surgical techniques were described, but all are associated with significant morbidity and mortality rates, especially in elderly patients with severe comorbidities. ${ }^{1}$

The first stent-grafting procedure in the human thoracic aorta was allotted to Volodos and colleagues in $1991 .^{2}$ It quickly offered the promise of a new and less-invasive method of achieving aneurysm exclusion. ${ }^{3}$ A restricted number of groups demonstrated technical feasibility with perioperative morbidity and mortality rates lower than those encountered after conventional surgical treatment. ${ }^{4-12}$ Even so, the endovascular repair of aneurysms of the descending 
TABLE 1. Preoperative clinical characteristics of the 45 patients with descending thoracic aortic aneurysms treated with endovascular stent grafts

\begin{tabular}{lcc}
\hline Characteristics & No. of & \\
patients & $\%$ \\
\hline Operative status & & \\
Aspirin 1 & 2 & 4.4 \\
Aspirin 2 & 14 & 31.1 \\
Aspirin 3 & 13 & 28.9 \\
Aspirin 4 & 16 & 35.5 \\
Emergent & 8 & 17.8 \\
Non emergent & 37 & 82.2 \\
Other medical problems & & \\
Coronary artery disease & 24 & 53.3 \\
Congestive heart failure & 19 & 42.2 \\
Renal insufficiency & 10 & 22.2 \\
Hypertension & 34 & 75.6 \\
Diabetes mellitus & 12 & 26.7 \\
Dyslipidemia & 9 & 20 \\
Stroke & 4 & 8.9 \\
Cigarette smoking & 27 & 60 \\
Chronic pulmonary disease & 26 & 57.8 \\
Previous cardiac, thoracic, or vascular surgery & 11 & 24.4 \\
\hline
\end{tabular}

thoracic aorta is less widespread than procedures carried out on the infrarenal aorta. The long-term results are thus not well known.

The objective of this study is to report the midterm results of endovascular repair of aneurysms of the descending thoracic aorta with the exclusive use of second-generation stent grafts.

\section{Patients and Methods}

\section{Patients}

From February 1996 through September 2005, 45 consecutive patients with an average age of $68 \pm 11$ years (range, 47-81 years) were included in a monocentric, nonrandomized, prospective study approved by our hospital ethics committee. Informed consent was obtained from all patients or patient families. All patients presented with an atherosclerotic aneurysm of the descending thoracic aorta. Chronic or acute aortic dissections, penetrating ulcers, and traumatic ruptures were excluded. The preoperative clinical characteristics are summarized in Table 1. Stent grafting was performed in all cases.

\section{Preoperative Imaging}

All patients underwent spiral computed tomographic angiography scanning with 3-dimensional reconstruction before stent graft placement so as to determine the location, length, and diameter of the aneurysm and landing zones, as well as to evaluate the suitability of the iliac and femoral arteries for vascular access. The preoperative characteristics of the aortic lesions are summarized in Table 2.
TABLE 2. Preoperative characteristics of the aortic aneurysms

\begin{tabular}{|c|c|c|c|}
\hline \multicolumn{2}{|c|}{ Size of aneurysms } & $\begin{array}{c}\text { Mean } \pm \text { SD } \\
(\mathrm{mm})\end{array}$ & $\begin{array}{c}\text { Range } \\
(\mathrm{mm})\end{array}$ \\
\hline \multicolumn{2}{|c|}{ Aneurysm length } & $70 \pm 32.1$ & $30-130$ \\
\hline \multicolumn{2}{|c|}{ Aneurysm diameter } & $67 \pm 18$ & $40-100$ \\
\hline \multicolumn{2}{|c|}{ Proximal neck diameter } & $31 \pm 4.5$ & $24-39$ \\
\hline \multicolumn{2}{|c|}{ Distal neck diameter } & $31.5 \pm 3.9$ & $24-40$ \\
\hline \multicolumn{2}{|c|}{ Distance from the left subclavian artery } & $39.2 \pm 27.7$ & $0-130$ \\
\hline Location & No. of patients & \multicolumn{2}{|c|}{$\%$} \\
\hline TAA 1 & 11 & \multicolumn{2}{|c|}{46.6} \\
\hline TAA 2 & 6 & \multicolumn{2}{|c|}{13.3} \\
\hline TAA 3 & 14 & \multicolumn{2}{|c|}{31.1} \\
\hline TAA 4 & 1 & \multicolumn{2}{|c|}{2.2} \\
\hline
\end{tabular}

$S D$, Standard deviation; TAA, thoracic aortic aneurysm.

Intravascular Device Details, Stent Graft Placement, and Postoperative Care

All intravascular devices used were covered stent grafts: 17 Excluder devices (W. L. Gore \& Associates, Elkton, Md), 27 Talent devices (Medtronic, Inc, Minneapolis, Minn), and 1 Baxter device. Stent graft dimensions were oversized by $10 \%$ to $15 \%$ compared with the landing zone diameters and were at least 30 to $40 \mathrm{~mm}$ longer than the aortic lesion to be treated. All the patients received an intravenous bolus of heparin at the dose of $75 \mathrm{UI} / \mathrm{kg}$. The patients were extubated immediately after the procedure and then supervised in the intensive care unit during the first 24 hours. Anticoagulation was maintained for 48 hours (preventive doses of low-weight heparin or calcic heparin according to the blood creatinin level) and then followed by aspirin $(250 \mathrm{mg} / \mathrm{d})$.

\section{Follow-up}

Follow up consisted of standard chest roentgenography, transesophageal echocardiography, and injected spiral scanner or magnetic resonance before discharge and on the 3rd, 6th, 12th, and 18th months. Standard chest roentgenography and injected spiral scanner or magnetic resonance were performed every subsequent year. One patient was lost from follow-up (2.2\%). Mean follow-up was $24.7 \pm 21.6$ months (range, 6 months- 6.7 years).

\section{Definitions}

The definitions used in this study are described in the literature. ${ }^{13}$ Primary technical success was defined as a complete exclusion of the aneurysm without an additional procedure and without perioperative death or complication. Secondary technical success was defined as a complete exclusion of the aneurysm after an additional endovascular procedure or after spontaneous thrombosis of primary endoleak within the first month. Endoleak was defined as the angiographic, tomodensitometric, or echocardiographic description of blood flow apart from the stent graft. Endoleaks were then classified according to the literature. ${ }^{14,15}$ Any perioperative deaths, any primary or secondary endoleaks, any failures of the endovascular device, any reinterventions, any deaths related to an aortic rupture, or any sudden unexplained late deaths were considered as failures of the treatment. 


\section{Statistical Analysis}

All the statistical studies were carried out with the STATA program (Stata Corp, College Station, Tex). The continuous variables were expressed as means \pm standard deviations.

The evaluation of actuarial survivals was carried out according to the Kaplan-Meier nonparametric method. The actuarial rates of survival were reported as $1 \pm$ standard deviation.

The multivariate study was performed by using the log-rank test, and the factors analyzed as potential predictors of adverse events were the age and sex of the patient, aspirin status, comorbidities (hypertension, diabetes mellitus, previous cerebrovascular accident, coronary disease, renal failure, and chronic pulmonary obstructive disease), emergency operation, location of the aneurysm, locations of the proximal and distal landing zones and their diameters, maximal diameter of the aneurysm, number of stent grafts used, covered length, and coverage of the T9-L1 intercostal arteries.

\section{Results \\ Periprocedural Outcome}

Briefly, all accesses were at the level of the underinguinal, iliofemoral junction. An average of $1.2 \pm 0.5$ stent grafts were used (from 1-3). The ostium of the left subclavian artery had to be covered in $6(13.3 \%)$ patients. The T9-T11 portion of the distal part of the descending thoracic aorta was covered in 16 cases ( $35.5 \%$ of the patients). No deaths occurred, and no conversion to open surgical intervention was required during the procedures. One rupture of the external iliac artery was encountered and required an iliofemoral bypass, which was performed in the same operative period.

\section{Early Outcome}

Mortality. Three patients died during the 30 days after the procedure. The causes of the deaths were 2 pneumoniae (days 6 and 28) and 1 aortoesophageal fistula, which was a consequence of the aneurysm before stent grafting (day 30). The perioperative death rate was $6.7 \%$. The death rate related to the treated aortic disease was $2.2 \%$. The death rate at 30 days of the patients treated in emergency situations was $12.5 \%$. It was $5.4 \%$ for the patients electively treated $(P>.05)$.

Morbidity. In the first 30 days after the intervention, 20 complications were encountered in 15 patients. Five patients presented with vascular complications: 2 with ischemia of the left upper limb, 2 with pseudoaneurysms of the left brachial artery, and 1 with rupture of the iliac artery. Nine patients presented with neurological complications. Cerebrovascular accidents were encountered in 6 patients, and this was the main complication $(13.3 \%)$. Of the 6 patients with their left subclavian artery covered, one patient presented with an ischemic left frontoparietal cerebrovascular accident. In all the other cases, the encephalic injuries were diffusely positioned intracerebrally and were also consistent with the manipulation of intravascular devices in an atherosclerotic aortic arch. Two (4.4\%) patients presented with paraplegia, and one of them had delayed paraplegia caused by a rupture of an external iliac artery with collapse. One patient presented with a transient left recurrent laryngeal nerve paralysis, which was identified during postoperative fiberoptic laryngeal examination. Two patients presented with severe respiratory complications and died from pneumonia.

Endoleaks, aortic complications, and reinterventions. Nineteen $(42.2 \%)$ primary endoleaks were encountered: thirteen (28.8\%) type I endoleaks, 1 (2.2\%) type II endoleak, and 5 (11.1\%) type III endoleaks. The primary technical success rate was $58.8 \%$. Two secondary endoleaks ( 1 type I and 1 type III) were observed, both within the first 30 days (4.4\%).

Aortic complications occurred in 4 patients. These complications were always associated with primary or secondary endoleaks. One contained rupture was associated with a secondary type III endoleak (day 23; Figure 1, $A$ and $B$ ). Three fistulae were associated with type I endoleak. One patient died on postoperative day 30 with a de novo aortobronchial fistula (primary type I endoleak). One aortoesophageal fistula (primary type I endoleak) and 1 aortobronchial fistula (secondary type I endoleak), which were both consequences of the aneurysm before stent grafting, recurred on postoperative days 9 and 31, respectively, and required reintervention.

Four patients underwent endovascular reintervention during the first month (2 primary type I [days 9 and 29], 1 primary type III [day 21], and 1 secondary type III [day 23] endoleaks). Three patients underwent endovascular reintervention because of the persistence of type I primary endoleaks in 2 cases (days 37 and 47) and because of a secondary type I endoleak in 1 case (day 31 ). The other primary endoleaks spontaneously thrombosed after 1 month. The secondary success rate was $97.8 \%$.

Shrinkage of the aneurysmal sac. During the first month, the aneurysmal diameter increased in 3 cases. In 2 cases the increase in diameter was transitory $(5$ and $10 \mathrm{~mm})$ without detectable endoleak, and in 1 case an increase in diameter of $30 \mathrm{~mm}$ was observed. This latter case was associated with a primary type I endoleak in a patient with 2 major postoperative complications (cerebrovascular accident and renal insufficiency), thus preventing the performance of reintervention. The patient died on postoperative day 30, with no reintervention having been performed. In all the other cases, the aneurysms shrank. The mean shrinkage of the aneurysmal sac was $12.8 \pm 10.3 \mathrm{~mm}$ at the end of the first month.

\section{Midterm Outcome}

No device failure, no migration, no kink or twist, and no secondary endoleak were observed beyond the 30th day after the procedure. No aortic reintervention was required 

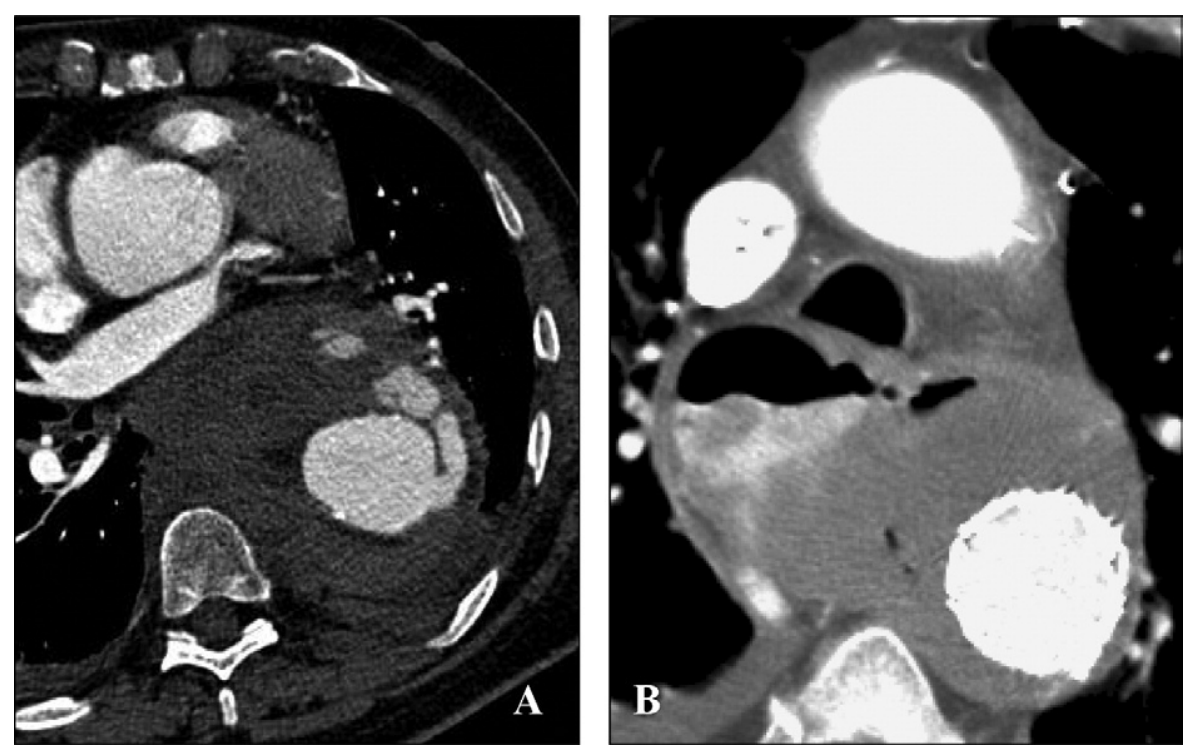

Figure 1. A and B, Spiral computed tomogram showing a rupture of the distal part of the descending thoracic aorta caused by a secondary type III endoleak 23 days after the procedure.

after the second month. Six patients died beyond the 30th day after the procedure. The causes of the deaths were renal insufficiency 62 days after the procedure, digestive hemorrhage in a cirrhotic patient 1.1 years after the procedure, myocardial infarction 1.8 years after the procedure, and cerebrovascular accident 3.1 years after the procedure. Two patients died suddenly 1 and 1.6 years after the procedure without any obvious cause having been highlighted and without any autopsy having been performed.
Survival Analysis

Actuarial survival estimates 1,3 , and 5 years after the procedure were $87.6 \% \pm 5.3 \%, 76.9 \% \pm 7.4 \%$, and $70.6 \% \pm$ $9.2 \%$, respectively (Figure $2, A$ ). More specifically, actuarial freedom from death related to the treated aortic disease was $94.3 \% \pm 4.0 \%, 94.3 \% \pm 4.0 \%$, and $86.4 \% \pm 8.4 \%$ at 1,3 , and 5 years, respectively (Figure 2, B). Actuarial freedom from complication was $62.6 \% \pm 7.7 \%, 58.9 \% \pm$ $8.1 \%$, and $58.9 \% \pm 8.1 \%$ at 1,3 , and 5 years, respectively (Figure 2, C).
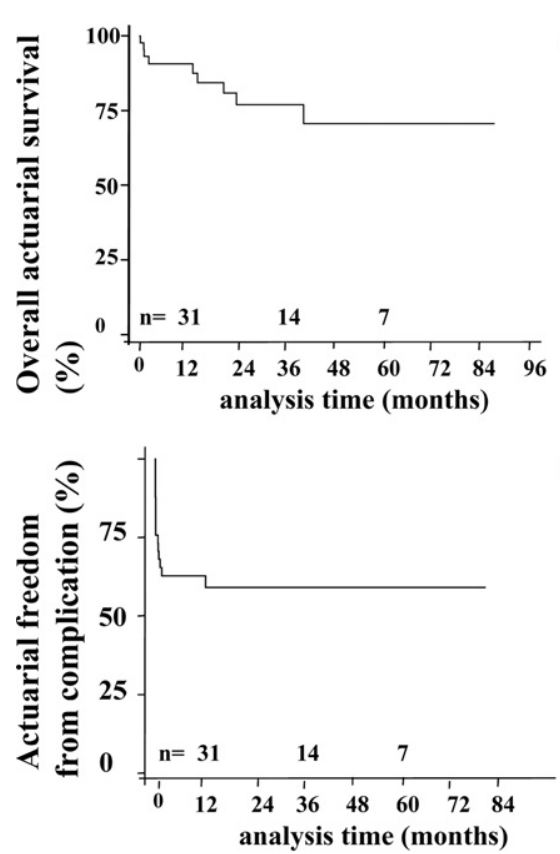

A

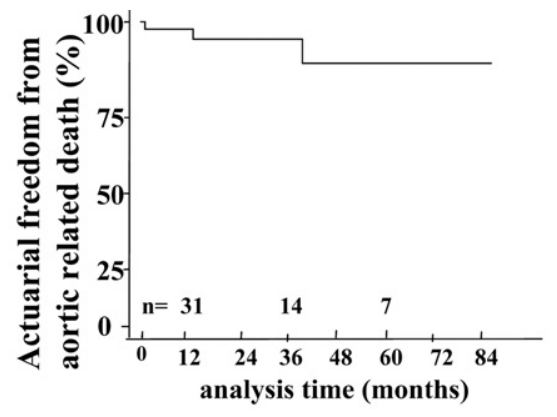

C

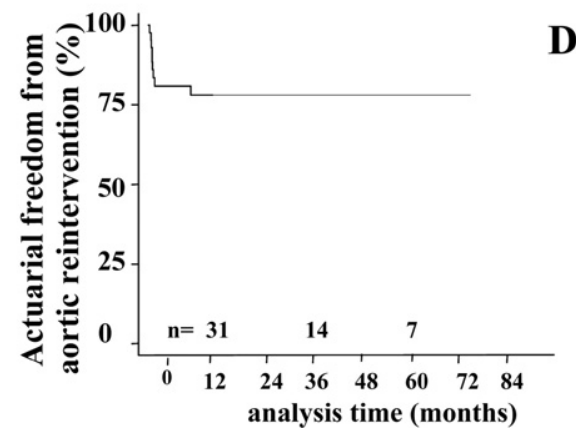

B

Figure 2. A, Overall actuarial survival estimates. B, Actuarial freedom from aortic-related death (see text for definition). C, Actuarial freedom from complication. D, Actuarial freedom from aortic reintervention. 
Actuarial freedom from treatment failure was $90.9 \% \pm$ $6.1 \%, 84.8 \% \pm 8.2 \%$, and $84.8 \% \pm 8.2 \%$ at 1,3 , and 5 years, respectively. Actuarial freedom from reintervention at 1,3 , and 5 years was $71.8 \% \pm 6.9 \%$. Actuarial freedom from aortic reintervention at 1,3 , and 5 years was $77.9 \% \pm$ $6.5 \%$ (Figure 2, D). All the aortic reinterventions were performed during the first year.

\section{Multivariate Analysis}

We identified aspirin score equal to or greater than $3(P$ $=.005)$, high diameter of the aneurysms $(P=.007)$, more than 1 stent graft deployed $(P=.0001)$, and long covered lengths $(P=.02)$ to portend a significantly higher likelihood of late death.

We identified the location of the aneurysm $(P=.05)$ and a high diameter of the aneurysm $(P=.04)$ to portend a significantly higher likelihood of secondary endoleak. We identified the location of the aneurysm $(P=.01)$ and high diameter of the aneurysm $(P=.04)$ to portend a significantly higher likelihood of treatment failure $(P=$ $.01)$.

\section{Discussion}

Even in large-volume centers, morbidity from elective open surgical repair is significant, with paraplegia and 30-day mortality rates, respectively, ranging from $3 \%$ to $15 \%$ and $3 \%$ to $12 \% .{ }^{16,17}$ Endovascular therapies are considered an evolutionary step toward less-invasive treatments for thoracic aortic diseases. The morbidity and mortality associated with the endovascular repair of thoracic aortic aneurysms seem to be less than those of conventional operative treatment, despite the fact that many patients were referred to endovascular treatment because of their high surgical risks. ${ }^{12}$ However, in spite of recent advances, endovascular repair of aneurysms of the descending thoracic aorta remains challenging. Moreover, very little is known about the long-term results of such procedures. Except for the series from Stanford dealing with the use of first-generation stent grafts in 103 patients, no more than 4 other series with an average follow-up exceeding 2 years are reported in the literature. ${ }^{12,18,19}$ Most of the publications are a mix of different indications, making comparisons difficult. For these reasons, only atherosclerotic aneurysms of the descending thoracic aorta were considered for this study, the goal of which was to determine the midterm results of endovascular repair in this indication by using secondgeneration, commercially produced thoracic stent grafts.

Although Dake and coworkers, ${ }^{20}$ using homemade devices, reported 58\% femoral access and up to 30\% aortic approach, we were able to introduce the stent graft through an infrainguinal iliofemoral approach in all the cases. Nevertheless, rupture of the external iliac artery was encountered in 2 patients. In one case this rupture was associated with secondary paraplegia because of a hemodynamic col- lapse, thus underlining that these vascular complications might have severe consequences.

Accurate placement of the stent graft in the arch is one of the most challenging technical feats, and perioperative stent graft migration is reported to occur in $2 \%$ to $20 \%$ of cases. $^{21,22}$ We avoided this problem with lower blood pressure during deployment, and we did not convert any endovascular procedure to open surgical intervention, which is a major improvement compared with the $4 \%$ and $12 \%$ conversion rate, respectively, reported by Dake ${ }^{23}$ and Greenberg and associates. ${ }^{7}$ This can also probably be explained by the technical improvements of the devices used, allowing partial deployment and correct repositioning before complete dropping into place of the devices.

Early mortality is the most important criterion in appraising the results of thoracic aortic aneurysm treatment and in comparing endovascular repair with conventional open surgery. According to the literature, the 30-day mortality rates range between $0 \%$ and $15 \%$. $4,8,18,19,21,22,24-27$ We report a 30 -day mortality rate of $6.6 \%$. Two patients died of severe pneumonopathy (keep in mind that chronic pulmonary disease was the main comorbidity), and 1 patient presented with an aortic-related 30-day death.

Paraplegia is one of the most feared complications after repair of thoracic aneurysms. Although the endovascular repair of thoracic aortic aneurysms shows a promising reduction in operative morbidity compared with open surgery, the risk of spinal cord ischemia still exists. According to the literature, paraplegia occurs in $0 \%$ to $6.5 \%$ of cases. ${ }^{4,10,18,21,25,27-29}$ Two (4.4\%) of our patients presented with paraplegia, one immediately. The other patient presented with secondary paraplegia after delayed hemodynamic collapse. Despite appropriate medical treatment, including cerebrospinal fluid drainage, these patients did not recover from their deficit. Because of a limited number of events, we were unable to identify any risk factors for paraplegia.

As reported by other authors, ${ }^{4,10,18,21,25,27-29}$ the main postoperative complication of endovascular repair of aneurysms of the descending thoracic aorta was cerebrovascular accident (13.3\%). Because the encephalic injuries were diffusely positioned, the occurrence of a cerebrovascular accident can easily be explained by the manipulation of wires and catheters in an atherosclerotic aortic arch. We try to minimize the risk of embolic stroke by limiting the manipulation within the aortic arch, particularly when severe atherosclerotic aortic arches are identified: we avoid handling the guides as much as possible at the level of the aortic valve and of the arch, a single guide wire is placed proximally to the aneurysm, we always maintain the rigid part of the guide wire upstream of the dropping device to decrease friction on the wall, and minimal progression of the undeployed stent graft is performed across the arch. 
Primary endoleak rates are similar to those encountered in the infrarenal aorta (4.2\% to $53 \%) .{ }^{23}$ Forty-two percent of our patients experienced primary endoleak, identified at the end of the procedure by means of angiography, transesophageal echocardiography, or both. We observed type I (28.8\%) and type III (11.1\%) endoleaks and 1 type II endoleak related to an intercostal artery. Contrary to Greenberg and associates, ${ }^{7}$ we do not conclude that the presence of a primary endoleak should always be treated aggressively because most of the primary type I endoleaks (7/13) spontaneously thrombosed during the first month. Of 13 primary type I endoleaks, 7 spontaneously healed during the first month, and 4 required reintervention with a good result. One patient with an aortoesophageal fistula died, and another with an increase of diameter died because of another complication preventing the performance of reintervention. In fact, numbers of type I endoleaks are minor (mostly when identified on transesophageal echocardiography) and spontaneously heal, provided that the choice of the diameter of the stent graft and its positioning are optimal. In these cases we are content to model the prosthesis with a balloon to achieve optimal proximal seal. In case of an evident error in the diameter or in the positioning of the device, it is necessary to consider the use of a proximal long extension with a higher diameter (and not a cuff, which would not present a sufficient overlapping). If the endoleak persists, even remotely, it would be necessary to perform reintervention. Two patients experienced secondary endoleaks, 1 type I and 1 type III, during the first postoperative 30 days. Concerning the secondary type I endoleak, the proximal neck was probably too short, whereas no technical failure was identified in the secondary type III endoleak. Both of the secondary endoleaks were associated with aortic complications. No secondary endoleak was observed beyond postoperative day 30 .

Despite a longer follow-up time than most of the references in the literature, we did not observe any increased long-term complication rate. Six patients died, but only 2 deaths were potentially related to the treated aortic disease (2 unexplained sudden deaths).

\section{Limitations of Our Study and Current Prospects}

Our work was prospective but monocentric and not randomized. Moreover, the number of complicating events and the total number of patients in the study made statistical analysis challenging. In particular, we could not identify any factor influencing the occurrence of paraplegia or endoleak. The absence of late aortic complications is encouraging but might only be related to follow-up duration, which remains short. Longer follow-up remains necessary, and we cannot exclude the occurrence of more delayed complications because of the evolution of the aortic morphology. ${ }^{30}$ Controlled studies comparing conventional surgery and endovascular repair in good surgical candidates and optimal medical treatment versus endovascular repair in bad surgical candidates would also be interesting. From a technical point of view, even if only very few consequences of primary endoleak were observed, we tend to reduce their occurrence rate through a more rigorous selection of the patients. This selection should not only be based on physiologic criteria but also on anatomic criteria. Moreover, we tend to choose longer proximal and distal landing zones, and we tend to perform more and more hybrid procedures associating extra-anatomic bypasses and endovascular repair.

\section{Conclusions}

Actually, short-term morbidity and mortality rates from endovascular treatment compare favorably with those from surgical intervention, and stent graft placement is proving to be a safe, minimally invasive, and effective treatment for atherosclerotic aneurysm of the descending thoracic aorta. However, the incidence of early and late complications highlights current challenges and underlines the necessity of close follow-up. Taking into account the gravity of the natural evolution without medical treatment and the serious and invasive nature of surgical treatment, endovascular repair of aneurysms of the descending thoracic aorta seems to have a central place among selected patients at high surgical risk. Nevertheless, equivalence or superiority of endovascular repair compared with conventional open surgical treatment for patients at low surgical risk has not been proved yet. The potential long-term risks of prosthetic degradation, migration, or surgical conversion remain unknown, as do their effects on survival. Further studies are still required to determine the most relevant indications and the long-term efficacy and durability of this innovative technique.

We acknowledge the kind help of Mrs Barbara Hollington in the preparation of the revised version of this manuscript.

\section{References}

1. Kouchoukos NT, Dougenis D. Surgery of the thoracic aorta. $N$ Engl J Med. 1997;336:1876-88.

2. Volodos NL, Karpovitch IP, Troyan VI. Clinical experience of the use of self-fixing synthetic prostheses for remote endoprosthetics of the thoracic and the abdominal aorta and iliac arteries through the femoral artery and as intraoperative endoprosthesis for aorta reconstruction. Vasa Suppl. 1991;33:93-5.

3. Dake MD, Miller DC, Semba CP, et al. Transluminal placement of endovascular stent-grafts for the treatment of descending thoracic aortic aneurysms. $N$ Engl J Med. 1994;331:1729-34.

4. Ehrlich M, Grabenwoger M, Cartes-Zumelzu F, et al. Endovascular stent graft repair for aneurysms on the descending thoracic aorta. Ann Thorac Surg. 1998;66:19-25.

5. Mitchell RS, Miller DC, Dake MD, et al. Thoracic aortic aneurysm repair with an endovascular stent graft: the "first generation." Ann Thorac Surg. 1999;67:1971-4. 
6. Grabenwoger M, Hutschala D, Ehrlich MP, et al. Thoracic aortic aneurysms: treatment with endovascular self-expandable stent grafts. Ann Thorac Surg. 2000;69:441-5.

7. Greenberg R, Resch T, Nyman U, et al. Endovascular repair of descending thoracic aortic aneurysms: an early experience with intermediate- term follow-up. J Vasc Surg. 2000;31:147-56.

8. Criado FJ, Clark NS, Barnatan MF. Stent graft repair in the aortic arch and descending thoracic aorta: a 4-year experience. J Vasc Surg. 2002;36:1121-8.

9. White RA, Donayre CE, Walot I, et al. Endovascular exclusion of descending thoracic aortic aneurysms and chronic dissections: initial clinical results with the aneuRx device. J Vasc Surg. 2001;33: 927-34.

10. Orend KH, Scharrer-Pamler R, Kapfer X, et al. Endovascular treatment in diseases of the descending thoracic aorta: 6-year results of a single center. J Vasc Surg. 2003;37:91-9.

11. Ellozy SH, Carroccio A, Minor M, et al. Challenges of endovascular tube graft repair of thoracic aortic aneurysm: midterm follow-up and lessons learned. J Vasc Surg. 2003;38:676-83.

12. Demers P, Miller DC, Mitchell RS, et al. Midterm results of endovascular repair of descending thoracic aortic aneurysms with first-generation stent grafts. J Thorac Cardiovasc Surg. 2004;127: 664-73.

13. Chaikof EL, Blankensteijn JD, Harris PL, et al. Reporting standards for endovascular aortic aneurysm repair. J Vasc Surg. 2002;35: 1048-60.

14. White GH, Yu W, May J, et al. Endoleak as a complication of endoluminal grafting of abdominal aortic aneurysms: classification, incidence, diagnosis and management. J Endovasc Surg. 1997;4: 152-68.

15. White GH, May J, Waugh RC, et al. Type III and type IV endoleak: toward a complete definition of blood flow in the sac after endoluminal AAA repair. $J$ Endovasc Surg. 1998;5:305-9.

16. Clouse WM, Hallett JW Jr, Schaff HV, et al. Improved prognosis of thoracic aortic aneurysms: a population-based study. JAMA. 1998;280: 1926-9.

17. Coselli JS, LeMaire SA, Conklin LD, et al. Left heart bypass during descending thoracic aortic aneurysm repair does not reduce the incidence of paraplegia. Ann Thorac Surg. 2004;77:1298-303.
18. Scharrer-Pamler R, Kotsis T, Kapfer X, et al. Complications after endovascular treatment of thoracic aortic aneurysms. J Endovasc Ther. 2003;10:711-8.

19. Czerny M, Cejna M, Hutschala D, et al. Stent-graft placement in atherosclerotic descending thoracic aortic aneurysms: midterm results. J Endovasc Ther. 2004;11:26-32.

20. Dake MD, Miller DC, Mitchell RS, et al. The "first generation" of endovascular stent-grafts for patients with aneurysms of the descending thoracic aorta. J Thorac Cardiovasc Surg. 1998;116:689-703.

21. Neuhauser B, Perkmann R, Greiner A, et al. Mid-term results after endovascular repair of the atherosclerotic descending thoracic aortic aneurysm. Eur J Vasc Endovasc Surg. 2004;28:146-53.

22. Tse LW, MacKenzie KS, Montreuil B, et al. The proximal landing zone in endovascular repair of the thoracic aorta. Ann Vasc Surg. 2004;18:178-85.

23. Dake MD. Endovascular stent-graft management of thoracic aortic diseases. Eur J Radiol. 2001;39:42-9.

24. Grabenwoeger M, Hutschala D, Ehrlich M, et al. Endovascular stentgraft repair for aneurysms of the descending thoracic aorta. Ann Thorac Surg. 1998;66:19-25.

25. Nienaber CA, Fattori R, Lund G, et al. Nonsurgical reconstruction of thoracic aortic dissection by stent-graft placement. $N$ Engl J Med. 1999;340:1539-45.

26. Czermak BV, Waldenberger P, Perkmann R, et al. Placement of endovascular stent-grafts for emergency treatment of acute disease. Am J Roentgenol. 2002;179:337-45.

27. Schoder M, Cartes-Zumelzu F, Grabenwoger M, et al. Elective endovascular stent-graft repair of atherosclerotic thoracic aortic aneurysms: clinical results and midterm follow-up. Am J Roentgenol. 2003;180: 709-15.

28. Najibi S, Terramani TT, Weiss VJ, et al. Endovascular aortic aneurysm operations. Arch Surg. 2002;137:211-6.

29. Bortone AS, De Cillis E, D’Agostino D, et al. Endovascular treatment of thoracic aortic disease: four years of experience. Circulation. 2004; 110(suppl):II262-7.

30. Hassoun HT, Mitchell RS, Makaroun MS, et al. Aortic neck morphology after endovascular repair of descending thoracic aortic aneurysms. J Vasc Surg. 2006;43:26-31. 